\title{
Global Software Processes Definition in a Distributed Environment ${ }^{1}$
}

\author{
Mariângela Vanzin*, Marcelo Blois Ribeiro*, Rafael Prikladnicki*, \\ Ilmari Ceccato, Dante Antunes \\ *Pontifícia Universidade Católica do Rio Grande do Sul-PUCRS \\ [mvanzin,blois,rafael]@inf.pucrs.br, \\ ilmari_ceccato@terra.com.br,antunesdantec@aol.com
}

\begin{abstract}
Global software development projects are passing through the boundaries of a company or a country. In order to manage global teams effectively, it is necessary to define a software process adapted to different organizational cultures. A global software process gives all team members a common language to define tasks and activities allowing the better understanding of the business domain terms and the project milestones in spite of their differences in terms of culture and organizational structures. This paper presents the practices adopted to define a global software process for a distributed environment and the factors that impacted in the process definition. Lessons learned are also presented.
\end{abstract}

\section{Introduction}

Software engineers have recognized the influence of business globalization, once the organizations have been using software as a competitive factor. Global software development means that the software development is disperse along several sites (software development centers) that could be located in different countries and even continents. A global software team executes the activities collaborating on a common software development project [1].

According to Herbsleb et al [2] several factors have accelerated the adoption of global software development practices: (1) global resource pool management to assure the successfully and cost-competitively resources usage, wherever located; (2) the business advantages of proximity to the market, including customers and local conditions; (3) the quick formation of global software teams to exploit market opportunities; (4) the possibility to take advantages of the time zone difference in "roundthe-clock" development; and (5) the need for flexibility to attend the opportunities wherever they are present.

Several companies, such as Lucent, IBM, Motorola, among others are adopting the global software development. According to Carmel et al [1], in 2001, 203 of the US Fortune 500 organizations were engaged in global software development. Moreover, more than 50 nations were participating in collaborative software development internationally.

Recent works describe the problems of global software development (e.g. [3]), including physical distance between the groups of developers; time-zone differences; communication problems among teams; cultural differences; lack of trust due to the physical distance; and others. To achieve success in global software development, the companies need to adapt their processes, tools, and organizational structure to minimize these problems.

When global production processes are analyzed, such as automobiles manufacturing, it is possible to notice that organizations decide to globalize their processes if their internal manufacturing processes are mature and ready to manage the dispersion complexity [4]. In software, however, it is clear that many organizations with immature software development processes are working in a global perspective. In order to manage a globally dispersed team effectively, a global software process should exist.

A process defines how a software should be produced, specifying for example, the artifacts developed along the process, the milestones, etc. A software process gives all team members a shared and consistent view in terms of

\footnotetext{
${ }^{1}$ Study developed by the Research Group on Globally Distributed Software Development of the PDTI program, financed by Dell Computers of Brazil Ltd. with resources of Law 8.248/91.
} 
tasks, activities, roles, terms and milestones involved in software development. A software process has other important benefits: it groups similar activities together; it reduces redundant activities and excessive work; it organizes activities into steps and phases; it enhances quality by assuring that the activities are comprehensive and complete; and it serves as effective documentation for management.

These benefits are clear when all sites accept a global software process previously defined. Otherwise, if each site has its own development process, the adoption of a single process must be carefully evaluated since the learning curve can impact the delivery of the system as well as its costs [5].

This paper analyzes a set of practices to guide the global software processes definition involving sites geographically distributed. These practices have been defined by an organization using an ad hoc manner. We have searched in the literature, but we didn't find some study proposing a methodology to develop a global software process in a distributed environment. This way, besides analyzing the practices, we also identify and analyze the aspects that impact in their execution.

The paper is structured as follows. Section 2 presents the practices used to define global software processes. Section 3 describes the application of these practices in a real case study involving two sites with the same software capability maturity level. Section 4 describes the evaluation of the practices and the analysis of the factors that caused impact in the global software process definition. Section 5 presents the related work. Finally, section 6 presents the lessons learned and section 7 presents the conclusions and future work.

\section{Global Software Processes Definition}

The practices analyzed in this work were created by an organization that has many geographically distributed sites working together in software development projects. These practices were defined to guide the Global Software Processes Definition (GSPD) in order to have some benefits by using a global software process followed by all distributed sites; to assure the software quality; and to attend a strategic goal imposed by the headquarters.

The GSPD intends to define not only the global software processes, but also the artifacts produced by these processes, such as templates, guidelines, documentation, procedures, and so on.

The GSPD involves a global team separated by a national boundary while actively collaborating to define a common software process. Many sites can compose the global team, and each team has a focal point, which is the team members' leader. Each site can assume the Process Owner role or the Process Reviewer role.

The Process Owner role is responsible for defining the global software process and its team members are physically working together in a specific site. To perform his responsibility, the Process Owner should consider the pre-existent processes, the global environment, the organizational needs and the guidelines for processes definition. The Process Owner is also responsible for updating the defined process according to the suggestions collected and discussed. The Process Reviewer comprehends the team members responsible for reviewing the artifacts and suggesting updates.

The practices assume that just one site plays the Process Owner role and one or more sites plays the Process Reviewer role. All the teams that play the Process Reviewer role must interact with the Process Owner team members responsible for managing the information.

The actors are represented in Figure 1 as the business actors in the UML business use case diagram.

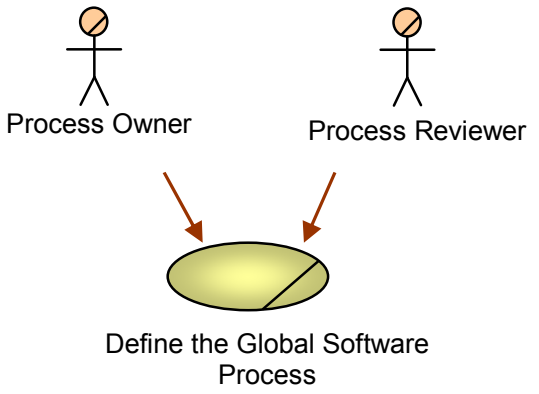

Figure 1. GSPD UML Use Case Diagram

The collaborative work involved in the process definition needs a common language definition for process formalization and teams' interaction. The communication resources (synchronous and asynchronous), such as teleconferencing facilities, also should be specified to support the interactions within the Process Owner and the Process Reviewer. The business use case "Define the Global Software Process" is detailed in the activity diagram depicted in

Figure 2.

The Process Owner plans the GSPD activities and defines the group deadlines. Then, the Process Owner schedules a kick-off meeting with the Process Reviewer team members. This meeting is central in global software process definition since it allows a) people to know each other; b) the whole group to discuss the GSPD schedule 
proposed by the Process Owner; c) team leaders to stimulate trust and commitment between the teams members; and d) teamwork synchronization.

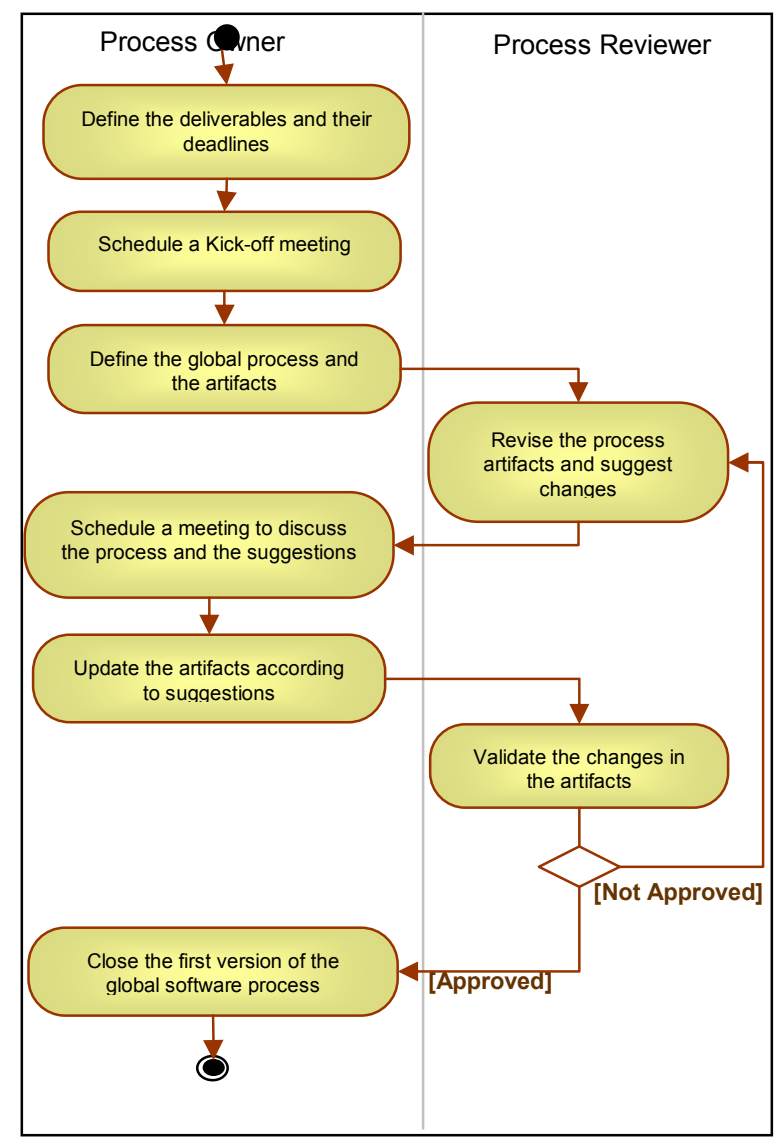

Figure 2. GSPD UML Activity Diagram

The Process Owner is responsible for defining the software process and the process artifacts. Frequent meetings with team members should be done to discuss and create the artifacts. The qualification of the team members is very important to assure the process quality. The team members should have knowledge about the organizational environment and the process area.

The first software process draft should be sent to the Process Reviewers. The dispersed team should review the artifacts proposed locally. The Process Reviewer analyze the software process draft with a critical view, verifying if it: a) is coherent with the organizational environment; b) addresses the organization needs; and c) is following the practices proposed by a software quality model.
According to the analysis done in the review meetings, the Process Reviewers create a process' change request documentation and send it to the Process Owner focal point (i.e. team coordinator). Then, the Process Owner schedules a meeting to discuss the process defined, to clarify some misunderstanding, and to explain the changes proposed. All the teams involved should agree with the process changes before its publication. Since many changes occur in this process, it is necessary to have a document version control procedure to manage the document updating.

The global process definition includes the creation of document templates related to the process execution. The Process Owner must maintain the consistency between the process itself and the templates used in its execution when changes are proposed.

Finally, if the process, the templates and the artifacts are coherent with the organizational needs, the Process Owner can publish the global software process in the organization Intranet. After the process definition activities, it is necessary to institutionalize the GSP in different sites. The institutionalization policies are not the focus of this paper. But the idea is that the Process Owner should develop a common training material and each site is responsible for training the employees.

\section{Case Study}

This research is exploratory in nature and based on case study [6]. The case study was developed in an organization focused in global software development. The data collection (research instrument) and data analysis are described in the section 4.

The practices described for GSPD were used by two sites that composed the global team. Both sites were distant geographically: one was located in South America and the other in Asia. In this paper, we refer the first one as Site A and to the other one as Site B.

Site A and B intend to achieve the third maturity level defined by the Capability Maturity Model (CMM) [7]. CMM defines levels of process improvement in software development. Each succeeding level indicates an improvement in the institutionalization of project management and software development methodologies within an organization.

Many processes must be defined to achieve CMM level 3. In this particular case study, the proposed practices were used to specify 12 processes. Many teams (Process Owner and Reviewers) in Site A and B were defined to work together in GSPD. People involved in 
the processes definition were defined by the global SEPG (Software Engineering Process Group) and grouped in teams of 3 to 5 members. Each site has its own SEPG and there are some representatives composing the global SEPG.

The processes goals and the roles assumed by each site are summarized in the Table 1.

Some aspects were considered to implement the GSPD activities. They are:

- The conference call infrastructure was used to assure the synchronous communication between the team members and the e-mail was used to any other needs, such as documents transmission, meetings appointments and questions answering;

- In order to control the document version, each document has a particular section to track the document updating and versioning;

- The English language was defined as the common language for all communications.

The GSPD practices were implemented according to the activities described in

Figure 2 .

Table 1. Processes defined

\begin{tabular}{|c|c|c|}
\hline Process Focus & $\begin{array}{l}\text { Process } \\
\text { Owner }\end{array}$ & $\begin{array}{c}\text { Process } \\
\text { Reviewer }\end{array}$ \\
\hline $\begin{array}{l}\text { Project Estimation and } \\
\text { Scheduling }\end{array}$ & Site A & Site B \\
\hline Project Management & Site B & Site A \\
\hline Requirements Management & Site B & Site A \\
\hline Risk Management & Site A & Site B \\
\hline Organizational Training & Site A & Site B \\
\hline Software Test & Site A & Site B \\
\hline Software Design & Site A & Site B \\
\hline Code and Unit Test & Site A & Site B \\
\hline Peer Review & Site B & Site A \\
\hline Configuration Management & Site B & Site A \\
\hline Software Quality Assurance & Site B & Site A \\
\hline Metrics & Site B & Site A \\
\hline Software Process Improvement & Site A & Site B \\
\hline
\end{tabular}

\section{Impact Factors in GSPD}

\subsection{Survey}

A systematic and rigorous approach is required to develop measuring instruments. Intending to evaluate the GSPD practices and the factors that have caused impact in the global activities, we defined a survey that specified measurements items.
The survey was divided into two parts. The first part identifies the aspects that have caused positive and negative impact on GSPD. These aspects were defined based on global software development literature (e.g [1$5,9]$ ) and were validated by the organization SEPG. They are: cultural differences, different time zone, English language, pre-existent processes, communication infrastructure, organizational structure, team trust, and team size.

The second part aimed to evaluate the practices used in GSPD. The questions defined intended to check: a) if the practices used to define the global process were adequate; b) if the cooperation between Site A and B was sufficient to assure the final product quality; c) if the communication infrastructure used was sufficient to achieve the goals; d) if the communication infrastructure would be better to improve the practices used; e) if the facilities used to control the document versioning and changing were sufficient; f) if different views between teams helped to improve the process quality; g) if the GSPD final product achieved the organization's expectation; and finally $h$ ) if the practices used in the GSPD could be reused to develop other processes to achieve others CMM maturity levels.

The survey was answered by 20 of 34 employees who have participated in GSPD in Site A. All items were measured through direct questions and using a threepoint Likert-type scale. In the first survey part, the scale considered was: a) $0=\mathrm{I}$ do not have knowledge to evaluate this question; b) 1 = the factor have caused a negative impact in GSPD; and c) $2=$ the factor have caused a positive impact in GSPD. In the second part, we also measured the items using the three-point Likerttype scale defined as: a) $0=I$ do not have knowledge to evaluate this question; b) $1=\mathrm{No}$; and c) $2=$ Yes.

The results analysis is described next considering two focuses: global factors that have caused impact in GSPD and the practices and environment used to GSPD. The Sphin $\mathrm{x}^{2}$ tool was used to generate statistics that supports the first data analysis section. The second data analyses section was done using the Intelligent Miner tool ${ }^{3}$ that permits to discover association rules between the global factors and the questions that evaluate the practices used in GSPD. The algorithm available in the tool, called Apriori, is described in [8].

\subsection{Global Factors}

\footnotetext{
${ }^{2}$ http://www.sphinxbrasil.com/

${ }^{3}$ http://www-306.ibm.com/software/data/iminer/
} 
The statistics related to global factors are given in Table 2. As showed, the global factors that have caused strong positive impact (considering an over $60 \%$ rate as a clear tendency) in GSPD are: the English language, the pre-existent processes, the communication infrastructure, the team trust, and the team size. Just the time zone difference has caused strong negative impact in GSPD. In order to understand how this impact ratings were achieved it is necessary to analyze each factor in detail.

Table 2. Global Factors Measurement

\begin{tabular}{|c|c|c|c|c|c|}
\hline & \multirow[b]{2}{*}{ Global Factors } & \multicolumn{4}{|c|}{ Statistics (\%) } \\
\hline & & $\mathbf{0}$ & 1 & 2 & $\begin{array}{l}\text { Not } \\
\text { answ } \\
\text { ered }\end{array}$ \\
\hline \multirow{9}{*}{ 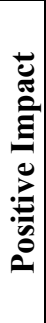 } & Cultural differences & 10 & 33 & 52 & 5 \\
\hline & Difference time zone & 29 & 57 & 14 & 0 \\
\hline & English language & 5 & 14 & 81 & 0 \\
\hline & Pre-existent processes & 14 & 10 & 76 & 0 \\
\hline & Communication & & & & \\
\hline & infrastructure & 14 & 24 & 62 & 0 \\
\hline & Organizational structure & 14 & 33 & 52 & 0 \\
\hline & Team trust & 10 & 5 & 81 & 5 \\
\hline & Team size & 19 & 10 & 71 & 0 \\
\hline \multirow{9}{*}{ 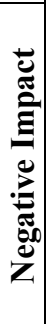 } & Cultural differences & 5 & 48 & 43 & 5 \\
\hline & Difference time zone & 19 & 14 & 62 & 5 \\
\hline & English language & 5 & 57 & 33 & 5 \\
\hline & Pre-existent processes & 29 & 38 & 29 & 5 \\
\hline & Communication & & & & \\
\hline & infrastructure & 14 & 52 & 29 & 5 \\
\hline & Organizational structure & 24 & 33 & 38 & 5 \\
\hline & Team trust & 19 & 43 & 29 & 10 \\
\hline & Team size & 14 & 62 & 19 & 5 \\
\hline
\end{tabular}

4.2.1. Cultural differences. According to Carmel [4], the culture provides members with images of their basic concerns, principles, ethics and bodies of manners, rituals, ideologies, strategies, and tactics of self-survival including certain notions of good deeds, various forms of folklore and legends, etc. The author describes two basic types of culture difference: nationality, the most obvious, and the organizational structure. National culture encompasses an ethnic group's norm, values and spoken languages, often delineated by the political boundaries of the nation state.

According to the Table 2, for 52\% of employees that have answered the survey, the cultural difference has impacted positively in the GSPD in order to exploit the benefits of views diversity within teams. The national culture diversity contributed to create a rich innovation environment appropriate for the processes definition. However, for $33 \%$ of employees that have answered the survey, cultural difference has impacted negatively because the center A have a different manner to work, e.g., they seams to give not much importance for some activities, such as to finish a deliverable in the planned date, to communicate a decision, etc. Other point mention were that the center A does not have any idea on how the center B reacts in front of some critics made by the center A. For example, they agree with the process, but the center $\mathrm{A}$ is not sure that the center B is following the process.

4.2.2. Time zone difference. Carmel et al [1] define temporal distance as the distance between the time zones in two different sites. Table 2 shows that the temporal distance (i.e., time zone difference) has caused a negative impact in GSPD. The temporal distance can be reduced using asynchronous and synchronous communication. If the temporal distance is great, as in this case study (12 hours), typically asynchronous technologies are used to support communication. The tasks execution has the advantages of a "follow-the-sun" kind of work. On the other hand, asynchronous communication is not so rich as synchronous communication: it does not convey information such as the speed and tone of voice, facial information, body language, pauses, etc. Therefore, synchronous communication is more effective and helps to solve conflicts faster. However, synchronous communication can be costly for employees who complain about the need to compromise personal life to have meetings with the distant colleagues. This latest issue was the main reason why Site A employees judged time zone negatively in the survey.

4.2.3. English language. One of the main problems in global software development is the language barrier, i.e., globally dispersed groups speak different languages.

In this case study, we have adopted the English language to reduce the national culture distance, considering that this language is not an official language of any site. All the artifacts were written in English and all the direct communication used the same language. English was chosen because the company has programs to motivate its employees to use English as the main language for written communication. This factor had a great influence in the survey results. A common language avoids misunderstandings between the team members.

4.2.4. Pre-existent processes. Before GSPD, both Process Owner and Reviewer had pre-existent processes being used in the local sites. These pre-existent processes have caused a positive impact in GSPD because they were used as an input to develop a new process. The Process Reviewer was focused on the discussion of 
divergences between the local processes in order to propose a new common process. In this scenario, the definition was done faster since people concentrated in the divergent points.

4.2.5. Communication infrastructure. There is a direct dependency among the temporal distance between the sites, the technology used for communication and the advantages that can be obtained with GSPD. Notice that both types of communication resources (synchronous and asynchronous) were used to support the activities described in

Figure 2. According to Table 2, the communication infrastructure has caused a positive impact to GSPD. It were used communication resources such as netmeeting, conference call, e-mail and instant messenger. These communication resources were sufficient to assure the team trust and commitment to achieve the GSPD goal.

4.2.6. Organizational structure. Organizational structure refers to the formal specification of different roles for organizational members, or tasks for groups, to ensure that the activities of the organization are carried out. Site A and B has some divergences in the organizational structure, e.g. some roles in Site B had not a correspondent one in Site A.

This divergence has caused a positive impact in the GSPD because the global teams should define general and flexible processes not restricted to a particular organizational structure. On the other hand, this organizational structure divergence has consumed an extra time to match the local roles to the global ones, but the effort dispended in this activity was not significant to cause a negative impact.

4.2.7. Team Trust. Trust is a recurrent problem in global teams. It is co-related with the poor communication among the team members due to the distance and the infrastructure used [1]. When trust increase between the teams, it impacts positively in the tasks, such as in GSPD. Site A teams trusted Site B teams because of the regular conference call meetings, enforcing the synergy among global team members. Another relevant factor was the strong experience in software development and process definition Site B team members have.

It is important to notice that this trust environment is derived from a progressive trust incentive policy. In this case study, both sites have been working together in other projects and the trust between team members had increased over time.
4.2.8. Team size. The Process Owner and Reviewer teams were composed by 3 to 5 employees. This team size was specified by the SEPG based on team size of previous local processes definition.

Considering the data showed in Table 2, the team size has impacted positively in GSPD. This size is suitable to collect different viewpoints while allowing a fast consensus and facilitating the team members' communication.

\subsection{Discovering Association Rules}

Intending to evaluate the practices used in GSPD and the global factors that have caused influence in these activities, we applied the algorithm defined by Agrawal et al [8]. It is a traditional mining technique that finds interesting associations and/or correlation relationships among a set of data items. Association rules show attributes value conditions that occur frequently together in a given dataset. Association rules provide information in the form of "if-then" statements. These rules are computed from the data and are probabilistic in nature.

An association rule is composed by and antecedent (the "if" part) and the consequent (the "then" part), and also two main measures: support and confidence. The support measure is simply the percentage of the number of transactions that include all items in the antecedent and consequent parts of the rule. The confidence is the ratio of the number of transactions that include all items in the consequent as well as the antecedent to the number of transactions that include all items in the antecedent.

In the GSPD context, each question value is an item used to compose an association rule. Each transaction list all items answered by an employee in a survey. Notice that questions are about the global factors and the practices used.

For example, the rule question- $f(2) \rightarrow$ question- $g(2)$ (support: $75 \%$, confidence $83,33 \%$ ) means that the $75 \%$ of employees have answered the question $f$ with value 2 and the question $g$ with value 2 . The confidence means that $83,33 \%$ of the employee that answer the question $f$ with value 2 , also answer the question $g$ with value 2 .

The data analyzed was restricted to the association rules with support higher than $50 \%$ and confidence higher than $80 \%$. If the support is low, it means that the rule is not worth to be considered or that it is simply less preferred (may be considered later). We consider 50\% as a representatively number of employees. 
4.3.1. Relation between the variables. In this subsection we are showing some interesting association rules discovered. One rule $(a+b \rightarrow c)$ means that $55 \%$ of the employees that considered the practices used in GSPD adequate (antecedent a) and the communication infrastructure sufficient to achieve the proposed goals (antecedent $b$ ), also understand that the cooperation was sufficient (consequent c). The rule confidence is $84.62 \%$. Thus, the cooperation between the teams was instigated by the practices defined (the teams should interact) and the adequate communication infrastructure used to support this interaction.

Analyzing another association rule $(d \rightarrow e)$, we verify that $75 \%$ of the employees who answered the survey think that knowledge and experience in CMM have caused positive impact (antecedent $d$ ); also considered that there was a trust between the teams (consequent $e$ ) with $83.33 \%$ of confidence. We conclude the CMM knowledge and experience contributed to trust development, because people are used to work on processes definition.

Other factor that contributes to develop a global software process is the convergence of different viewpoints. These different views were caused by the existence of pre-existence process, as proved by the rule $(f \rightarrow g)$ which indicates that $70 \%$ of the employees that think that the pre-existent processes have caused positive impact (antecedent $f$ ) in GSPD, also think that different views helped to define the global process (consequent $g$ ), considering $87.50 \%$ of confidence. No rule indicated that different viewpoints were related to the cultural difference, although we had supposed that this was the case.

As mentioned, different views between the teams have contributed to define a global process because of the different background and expertise of the team members. The problems related to different viewpoints convergence (consequence $g$ ) were minimized by the trust between the teams (antecedent $e$ ), as expressed by an association rule $(e \rightarrow g)$ with $50 \%$ of support and $90.91 \%$ of confidence.

Although the time zone difference caused a negative impact in GSPD, it did not influence the final project goal achievement because the practices used were appropriate to reduce its impact. It was also discovered in an association rule $(h \rightarrow i)$ that $50 \%$ of the employees know that the time zone have caused a negative (antecedent $h$ ) impact in the global software definition activities although the final goal was achieved (consequent $i$ ).
An association rule $(d+l+g \rightarrow i)$ related to the variable "final goal achieved" expresses that $70 \%$ of the employees thinks that CMM knowledge and experience (antecedent $d$ ), team size (antecedent $l$ ) and different views of the teams (antecedent $g$ ) caused positive impact in GSPD, also considered that the final product have achieved the organization goal and expectation (consequent $i$ ). This rule confidence is $100 \%$. Site B teams have a strong CMM knowledge and experience, and this is important to define the global process. The existence of different views about a certain topic also contributed to develop a generalized process intending to support distinct situations and environments. The team size also was a decisive factor in GSPD. The team size used was suitable for the goal proposed because the team size was big enough to permit the existence of different views between the team members, and also the team size was sufficient to allow the process definition considering distinct viewpoints.

Other relevant association rule $(a \rightarrow i)$ expresses the final GSPD goal was achieved because the practices used were adequate (antecedent $a$ ). This rule support is $70 \%$ and the confidence is $82.35 \%$. The practices used in GSPD supported the team interaction to define and update the global software processes.

4.3.2. Evaluating the practices used. Some association rules show interesting information about the practices used in GSPD. The Table 3 shows a set of association rules that have as a consequent if the practices used to define the global process were adequate. The rule antecedent, the support and confidence are specified in the table columns.

Trust between the team members is an important factor to assure the GSPD. As shown in the association rule number 1 , the practices were adequate to permit the trust development within the team members since they included a clear interaction support policy and a set of tools for that purpose. Another factor that contributed for Site A teams to develop trust in Site B teams was the strong CMM knowledge and experience. Carmel [4] calls swift trust to explain how individual who are "thrown together" quickly became productive in spite of not developing solid interpersonal relationship.

The practices defined also were sufficient to integrate the two sites that followed different software processes and had different points of view (rule 2 and 3 ). Thus, the practices have supported the teams' discussions to converge appropriately in a global software process.

As shown in the association rule 5 with $100 \%$ of confidence, the practices have permitted the teams' 
cooperation, being a fundamental factor to achieve the GSPD objectives. The practices proposed the interaction between the teams very often and also the clear definition of the roles assumed by each team.

The practices were also adequate in terms of the facilities to control de artifacts' versions and change requests (rule 4). The document version was controlled using a section in each artifact created. The change requests were specified using simple change request documentation. It stores a table composed by three columns. The first column specifies the name of the artifact the Process Review is requesting some changes. The second one describes the changes requested, and the last column should be filled by the Process Owner with information indicating if changes will be made or not.

Table 3. Associations Rules to evaluate the practices

\begin{tabular}{|l|l|c|c|}
\hline \multicolumn{1}{|c|}{ Rule Antecedent } & $\begin{array}{l}\text { Sup } \\
\text { (\%) }\end{array}$ & $\begin{array}{l}\text { Conf } \\
\text { (\%) }\end{array}$ \\
\hline $\mathbf{1}$ & $\begin{array}{l}\text { Team trust have caused positive Impact } \\
\text { Pre-existent processes have caused } \\
\text { positive Impact }\end{array}$ & 70 & 87.5 \\
$\mathbf{3}$ & $\begin{array}{l}\text { Different views between teams has } \\
\text { helped to improve the process quality }\end{array}$ & 80 & 88.8 \\
$\mathbf{4}$ & $\begin{array}{l}\text { The facilities used to control the } \\
\text { document version and changes } \\
\text { requested were sufficient }\end{array}$ & 70 & 100 \\
$\mathbf{5}$ & $\begin{array}{l}\text { The cooperation between A and B was } \\
\text { sufficient to assure the final product } \\
\text { quality }\end{array}$ & 70 & 93.3 \\
$\mathbf{6}$ & $\begin{array}{l}\text { The GSPD final product achieved the } \\
\text { organization's expectation } \\
\text { The practices used in the GSPD could } \\
\text { be reused to achieve others CMM } \\
\text { maturity levels }\end{array}$ & 80 & 88.8 \\
\hline
\end{tabular}

Finally, the rule 6 in the Table 3 means that the practices were adequate to achieve the goal proposed and for $80 \%$ of employees who participated in GSPD this method could be used to define other maturity levels proposed by the CMM (rule 7).

\section{Related Work}

Since it was not possible to identify similar approaches in the literature focusing on global process definition practices in distributed development environments, this section presents some works related to the global software development in a broader sense. Some of them identify problems related to global software development [1-5,9] such as delay in the software development process, lack of informal communication and cooperation across team, and others. These problems can be caused by many factors, such as cultural diversity, time zone difference, language issues, trust between the teams and so on. These studies were useful for our approach since we used them to analyze the impact of the proposed factors in the case study environment.

Other works (e.g. $[10,11])$ are focused on processcentered software engineering in order to extend the local scope of processes beyond a single organization frontier, by additionally supporting processes linkage to reflect the dependency of the components being development in the collaborative work. Our approach is based on the evaluation of some practices used to define software processes in a distributed environment.

\section{Lessons Learned}

The study conducted shows many characteristics of a global software definition strategy. In this section, we will present the lessons learned we found.

Lesson 1: The organization environment is a key factor when evaluating practices for global software process definition.

The organization environment has great influence in GSPD success. As organization environment we mean the current initiatives to promote trust between teams, to enhance the communication infrastructure, and to standardize a common language for document writing. The English language factor has contributed to the communication between distributed teams, once both centers have strong knowledge on this language and incentives for the language improvement. The trust development between teams was also an important factor to achieve the goal since the organization had already developed the trust between teams in previous common projects.

Lesson 2: When defining global software processes, many factors identified can be related to each other.

The definition of global software processes can lead to the identification of some problems based on the factors identified in this paper. For this reason, the organization needs to identify the relation between these factors and what people are able to participate in the process definition activity. For example, the trust between teams was associated to the team previous 
experience with distributed activities and the CMM knowledge. Considering this context, it is more helpful and less problematic to involve people that have CMM knowledge and had some previous experience in working with distributed teams. This approach can lead to fewer problems with trust between teams. The identification of these relations can increase the positive impact in GSPD.

Lesson 3: To define Global Processes it is necessary to have local SEPGs to coordinate local activities.

This work results showed that the practices involving SEPGs in each center responsible for coordinating GSPD locally is appropriate to achieve the common goal. The company can have a global SEPG, but in terms of process definition it is necessary to work with local coordinators adapted to the local culture and environment. The use of local SEPG can improve the teams' commitment to GSPD and enforce that the processes defined will be institutionalized. When institutionalizing the global process the local SEPGs should share the best practices globally with the other SEPGs.

Lesson 4: The GSPD practices can be reused to execute other process definition or work activities.

This paper described some practices used for an organization to define global software processes. The GSPD was treated as a joint project (multi-site project) internally in the organization. Because of this, these practices could be reused to define or upgrade other processes or even to execute joint software development daily activities.

\section{Conclusions and Future Research}

It is becoming harder to justify completing a software development project inside company walls. As the software community appreciates the economy of merging diverse development skills and domain expertise, and as communication media become more sophisticated, the cost and technology pressure more companies toward global software development. The global software development is leading researchers to acquire new knowledge and to be more interdisciplinary. This paper advances the knowledge in the global software development area by analyzing some practices to guide the global software process definition.
A global software process is very important to guide the software development in a distributed environment. The organizations can use the GSPD practices to: a) define a new software process and force the software process usage as a standard; b) define a software process based on the previous software processes in usage; and c) define guidelines to drive the distinct software processes in a high level. All the strategies are useful, but the organization should first define the purpose of having a global software team working together and then define the processes themselves.

According to the evaluation methodology used, the results demonstrated that the practices proposed were suitable for process standardization among different development sites. A global software process can help the organization to assure the software quality when it is developed in a distributed environment.

Due to the small number of surveys collected, the results cannot be easily generalized. In this phase, it can be adopted the analytical generalization principle, proposed by [6]. Planned follow up studies in this topic will extend the evaluation and the analysis of all practices defined by the GSPD considering other employees in different sites.

\section{References}

[1]Carmel, E. and Agarwal, R. (2001). Tactical approaches for alleviating distance in global software development. IEEE Software, 18(2):22-29.

[2]Herbsleb, J. D. and Moitra, D. (2001). Guest editors' introduction: Global software development. IEEE Software, 18(2):16-20

[3]Edwards, H. K. and Sridhar, V. (2003). Analysis of the effectiveness of global virtual teams in software engineering projects. In Proceedings of the 36th Hawaii International Conference on System Sciences.

[4]Carmel, E. (1999). Global Software Teams: Collaborating A cross Borders and Time Zones. Prentice-Hall.

[5]Battin, R. D., Crocker, R., Kreidler, J., and Subramanian, K. (2001). Leveraging resources in global software development. IEEE Softw., 18(2):70-77.

[6]Yin, R. K. (1994). Case study research: design and methods, Sage, USA.

[7]Mellon, C., Paulk, M.C., Weber, C.V., Curtis, B., and Chrissis, M. B. (1995). The capability maturity model: guidelines for improving the software process. AddisonWesley Longman Publishing Co., Inc.

[8]Agrawal, R., Imielinski, T., and Swami, A. (1993). Mining association rules between sets of items in large data4bases. In 
Proc. of the ACM SIGMOD Int'l Conference on Management of Data, pages 207-216, Washington D.C.

[9]Dafoulas, G. and Macaulay, L. (2002). Investigating cultural differences in virtual software teams. Electronic Journal on Information Systems in Developing Countries, pages 1-14.

[10]Scherer, D. (1998). A distributed framework for integrated software process and deployment support. Institut für Technische Informatik und Kommunikationsnetze (TIK).

[11]Murer, T. and Scherer, D. (1998). Organizational integrity: facing the challenge of the global software process. Institut für Technische Informatik und Kommunikationsnetze (TIK). 